\title{
VIABILIDAD DE POLEN EN UNA LÍNEA DE GIRASOL CULTIVADO, EN EL GIRASOL SILVESTRE (HELIANTHUS ANNUUS L. SSP. TEXANUS HEISER) Y EN SU DESCENDENCIA HÍBRIDA
}

\author{
Rosalinda Mendoza Villarreal ${ }^{1}$, M. Humberto Reyes-Valdés ${ }^{2}$, Carlos Espinosa \\ ZaPATA $^{3}$ y José Ángel Villarreal Quintanilla ${ }^{4}$
}

${ }^{1}$ Departamento de Ciencias Básicas, Universidad Autónoma Agraria Antonio Narro 25315 Buenavista, Saltillo, Coahuila

${ }^{2}$ Departamento de Fitomejoramiento, Universidad Autónoma Agraria Antonio Narro 25315 Buenavista, Saltillo, Coahuila

${ }^{3}$ Instituto Nacional de Investigaciones Forestales, Agrícolas y Pecuarias. CIRNOC Km. 4.5, Carretera Durango-Mezquital, Durango, Durango.

${ }^{4}$ Departamento de Botánica, Universidad Autónoma Agraria Antonio Narro

25315 Buenavista, Saltillo, Coahuila

\section{RESUMEN}

Con el propósito de evaluar la posible alteración en el desarrollo de los gametos masculinos en la progenie de un cruzamiento entre dos subespecies de girasol, se estudió la viabilidad del polen en la línea cultivada AN-3, así como en la subespecie silvestre Helianthus annuus ssp. texanus y en la descendencia híbrida resultante del cruzamiento CMS-AN-3 x H. annuus ssp. texanus. De las plantas híbridas, 95\% exhibieron fertilidad masculina, lo cual indica la presencia de alelos restauradores de la fertilidad en las poblaciones silvestres. La viabilidad de polen no mostró diferencias significativas entre el híbrido (91.5\%), el progenitor cultivado $(94.6 \%)$ y el silvestre $(95.2 \%)$. Los resultados sugieren que la viabilidad de polen es una característica mantenida en niveles altos tanto en las poblaciones silvestres como las cultivadas, y que no hay indicios de que la domesticación del girasol haya influido sobre este carácter. En lo que concierne al uso de material silvestre para el mejoramiento genético del girasol cultivado, la viabilidad de polen en los híbridos no es una barrera para el uso de poblaciones locales (sureste de Coahuila) de $H$. annuus ssp. texanus en programas de cruzamientos con fines de introducción de caracteres de plantas espontáneas a las variedades élite.

Palabras clave: girasol, Helianthus, viabilidad de polen. 


\section{ABSTRACT}

To evaluate possible developmental alterations in male gametes in the progeny of a sunflower inter-subspecific cross, pollen viability was studied in the cultivated line AN-3, the wild subspecies Helianthus annuus ssp. texanus, and in the hybrid from the cross CMS-AN-3 x H. annuus ssp. texanus. Among the hybrid plants, $95 \%$ of the individuals showed male fertility, thus indicating that the target wild populations contain male-sterility restoration alleles. Pollen viability did not show significant differences between the hybrid (91.5\%), the cultivated $(94.6 \%)$ and wild parents $(95.2 \%)$. Results suggest that pollen viability is a trait maintained at high levels in wild and cultivated sunflower populations, and that there is no indication that sunflower domestication had an effect on this character. Regarding the use of wild material to improve the cultivated sunflower, pollen viability is not a barrier to use local populations (southeast of Coahuila) of H. annuus ssp. texanus to introduce wild genetic material to elite varieties.

Key words: Helianthus, pollen viability, sunflower.

\section{INTRODUCCIÓN}

Se conocen nueve especies espontáneas de Helianthus con distribución en México. La mayoría de ellas se registra del norte del país, entre las cuales Helianthus annuus L. también se distribuye hasta la Península de Yucatán (González-Elizondo y Gómez-Sánchez, 1992). Además, están presentes los géneros Tithonia y Viguiera, ambos emparentados con Helianthus (Gómez y González, 1994). De aquí que México cuente con un reservorio importante de material genético nativo relacionado con el girasol cultivado, que puede aprovecharse con fines de mejoramiento.

Para el óptimo aprovechamiento de las plantas silvestres en términos de perfeccionamiento genético convencional, resulta deseable conocer su compatibilidad en cruzamientos con plantas cultivadas, en cuanto a la posibilidad de hibridación y características de fertilidad de la descendencia. Dada la sensibilidad de los gametos masculinos a las anormalidades citogenéticas, la semiesterilidad puede ser frecuentemente evaluada por procedimientos de tinción que determinan la calidad del polen (Stelly et al., 1990). Además de su importancia para el mejoramiento de las plantas basado en cruzas amplias, el conocimiento de la fertilidad de los híbridos a través de la viabilidad del polen contribuye al entendimiento de las barreras reproductivas 
entre especies y subespecies y a conocer el efecto biológico de la domesticación y el aislamiento reproductivo artificial sobre la evolución de dichas barreras.

En los estudios de viabilidad y fertilidad de polen en girasol se han utilizado diversos tipos de tinción. Algunos de ellos se basaron en colorantes nucleares como el carmín propiónico (Whelan, 1982); citoplásmicos como el Buffalo Black NBR (Jackson, 1988); o diferenciales como el método de Alexander (Alexander, 1969; Atlagic, 1996). Dichos procedimientos detectan el polen no abortivo, e indirectamente evalúan la viabilidad del mismo.

Seha encontrado una fuerte correlación negativa entre las fallas en apareamiento cromosómico en la meiosis y la viabilidad de polen, determinada a través de su tinción en el género Helianthus (Quillet et al., 1995). Esta relación trae como consecuencia una reducción de la viabilidad del polen en cruzas amplias, como es el caso del cruzamiento de $H$. annuus L. x H. laciniatus A. Gray, cuya descendencia híbrida tuvo $2.2 \%$ de polen fértil, comparado con $97 \%$ en el promedio de los progenitores (Jackson, 1988). En el híbrido H. tuberosus L. x girasol cultivado, la viabilidad de polen experimentó una reducción de más de $50 \%$ comparada con la observada en los progenitores (Atlagic et al., 1993). Por otro lado, a través de la segregación de marcadores de ADN y aloenzimas en una retrocruza proveniente de la hibridación $H$. argophyllus Torr. \& Gray x H. annuus L., Quillet et al. (1995) encontraron evidencia de tres regiones cromosómicas que controlan la viabilidad de polen.

Tomando en consideración la importancia del uso de plantas silvestres de girasol y la evaluación de su compatibilidad en cruzas con material cultivado, el presente estudio se planteó con el objetivo de estudiar la viabilidad de polen en la línea AN-3 de girasol cultivado ( $H$. annuus var. macrocarpus Ck11.), así como en la subespecie silvestre H. annuus ssp. texanus Heiser y en la descendencia híbrida entre ambos taxa.

\section{MATERIALES Y MÉTODOS}

Se utilizaron la línea CMS-AN-3 (androestéril) de girasol cultivado y su mantenedora (línea B, con fertilidad masculina), originadas en la Universidad Autónoma Agraria Antonio Narro, así como plantas silvestres de girasol del área de Saltillo y Ramos Arizpe, Coah. (Cuadro 1), las cuales fueron identificadas en el herbario ANSM del Departamento de Botánica de dicha universidad como Helianthus annuus ssp. texanus Heiser. Los aquenios de estos materiales fueron recolectados entre agosto y diciembre de 1996, cosechando cinco capítulos por 
Cuadro 1. Procedencia de las plantas silvestres de Helianthus annuus ssp. texanus de los municipios de Saltillo y Ramos Arizpe, Coahuila. En la ubicación se da el nombre de la calle o carretera, seguido del nombre del municipio entre paréntesis.

\begin{tabular}{|c|c|c|c|}
\hline Colecta & Ubicación & Coordenadas & $\begin{array}{c}\text { Altitud } \\
\text { (m s.n.m.) }\end{array}$ \\
\hline 1 & N. Ortiz Garza y V. Carranza (Saltillo) & $25^{\circ} 29^{\prime} \mathrm{N}, 100^{\circ} 59^{\prime} \mathrm{W}$ & 1525 \\
\hline 2 & $\begin{array}{l}\text { O. González y L. Echeverría Álvarez } \\
\text { (Saltillo) }\end{array}$ & $25^{\circ} 24^{\prime} \mathrm{N}, 100^{\circ} 58^{\prime} \mathrm{W}$ & 1653 \\
\hline 3 & $\begin{array}{l}\text { Carretera Saltillo-Monclova km } 22 \\
\text { (Ramos Arizpe) }\end{array}$ & $25^{\circ} 34^{\prime} \mathrm{N}, 101^{\circ} 03^{\prime} \mathrm{W}$ & 1441 \\
\hline 4 & $\begin{array}{l}\text { Carretera Saltillo-Monclova km } 32 \\
\text { (Ramos Arizpe) }\end{array}$ & $25^{\circ} 38^{\prime} \mathrm{N}, 101^{\circ} 06^{\prime} \mathrm{W}$ & 1250 \\
\hline 5 & I. López Zertuche y N. Ortiz Garza (Saltillo) & $25^{\circ} 30^{\prime} \mathrm{N}, 100^{\circ} 59^{\prime} \mathrm{W}$ & 1550 \\
\hline 6 & $\begin{array}{l}\text { N. Ortiz Garza y L. Echeverría Álvarez } \\
\text { (Saltillo) }\end{array}$ & $25^{\circ} 26^{\prime} \mathrm{N}, 100^{\circ} 58^{\prime} \mathrm{W}$ & 1564 \\
\hline 7 & $\begin{array}{l}\text { L. Echeverría Álvarez y J. Valdés Sánchez } \\
\text { (Saltillo) }\end{array}$ & $25^{\circ} 26^{\prime} \mathrm{N}, 100^{\circ} 58^{\prime} \mathrm{W}$ & 1586 \\
\hline 8 & L. Echeverría Álvarez y F. J. Mery (Saltillo) & $25^{\circ} 24^{\prime} \mathrm{N}, 100^{\circ} 59^{\prime} \mathrm{W}$ & 1673 \\
\hline
\end{tabular}

individuo de al menos diez plantas en cada lugar de muestreo. Dichos frutos se depositaron en el banco de germoplasma silvestre local de $H$. annuus y Tithonia tubaeformis (Jacq.) Cass. que mantiene el Laboratorio de Análisis de Genomas Vegetales de la Universidad Autónoma Agraria Antonio Narro.

Los cruzamientos se realizaron entre la línea CMS-AN-3 y ocho individuos silvestres, en el campo experimental de Buenavista, Saltillo, Coahuila, durante el verano de 1997. Para ello se sembraron semillas de las plantas espontáneas en vasos de polipropileno dentro del invernadero y en estado de plántula se transplantaron al campo, mientras que las de la línea CMS-AN-3 se sembraron directamente en el suelo. Los materiales silvestres se iniciaron bajo techo porque su ciclo es más largo y requerían de un establecimiento temprano. Por otro lado, los aquenios de las poblaciones espontáneas son muy pequeños y con baja tasa de germinación, lo que hace impráctica su siembra directa en el campo con fines experimentales. Otra razón para usar previamente el almácigo en el material silvestre, es que existen plantas autóctonas de $H$. annuus en el campo experimental empleado que pueden dar lugar a errores en la interpretación de los resultados. 
Se cubrieron los botones florales con bolsas de papel y posteriormente se recolectó el polen de cada planta silvestre en cajas de Petri para fecundar los capítulos de la línea androestéril CMS-AN-3 previamente aislados con envolturas. Se realizaron tres polinizaciones a cada capítulo, con el uso de aplicadores de franela, evitando la contaminación por polen extraño. Para cada cruzamiento se emplearon siete plantas por colecta silvestre como polinizadores, y un promedio de siete individuos de la línea cultivada como progenitores hembra.

En el ciclo primavera-verano de 1999 se sembraron semillas de los progenitores cultivados y silvestres, así como de su descendencia híbrida, para llevar a cabo el estudio de polen. En lugar de la línea androestéril (CMS-AN-3) que no produce polen, se utilizó su estirpe análoga fértil (mantenedora). Un total de 15 botones de cada una de tres plantas de cada procedencia silvestre e híbrida se cubrieron con bolsas de papel. Las colectas de polen fresco se hicieron en cajas de Petri al amanecer. El polen obtenido de cada capítulo se mezcló para su homogeneización y se analizó en dos repeticiones. De esta manera, se examinó un promedio de 90 muestras por cada colecta de material silvestre y por cada híbrido. Por lo que respecta a la línea cultivada, ya que es genéticamente homogénea y presenta un capítulo por planta, se analizaron dos muestras por cada inflorescencia única de un total de 15 individuos.

La estimación de polen viable (no abortivo) se hizo con la técnica descrita por Jackson (1988), basada en el uso del colorante citoplásmico Buffalo Black NBR. Para ello se preparó una solución con $0.01 \%$ de colorante y $45 \%$ de ácido acético, en agua destilada. Por cada repetición se colocó una gota del líquido indicador, sobre la cual se aplicó una pequeña cantidad de polen con pinzas de punta, se dispersó con un cubreobjetos y se dejó reposar 20 segundos. Se realizaron conteos al microscopio de 100 granos de polen, para estimar el porcentaje de viabilidad. Se tomó como viable (no abortivo) el grano teñido de color azul intenso y como no viable el que sólo se coloreó ligeramente (Fig. 1).

Para comparar taxa, colectas y cruzamientos, se empleó en cada caso un modelo con submuestreo (Steel y Torrie, 1960):

$$
Y_{i j k}=u+T_{i}+E_{j(i)}+\delta_{k(i j)}
$$

donde:

$$
\begin{aligned}
u & =\text { media general } \\
i & =1,2, \ldots, t \text { (tratamientos: taxa, colectas o cruzamientos) } \\
j & =1,2, \ldots, p \text { (plantas dentro de tratamientos) } \\
k & =1,2, \ldots, m \text { (muestras dentro de plantas) }
\end{aligned}
$$




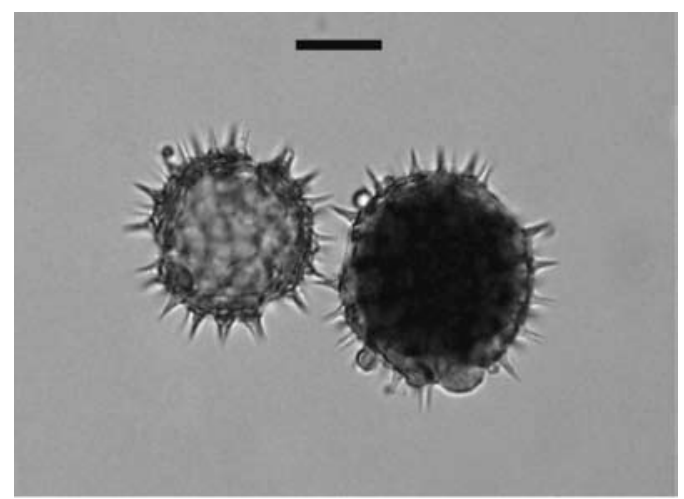

Fig. 1. Tinción diferencial con Buffalo Black NBR en dos granos de polen de girasol cultivado. El grano de polen del lado izquierdo es considerado como inviable, y el del lado derecho como viable. La escala en la parte superior equivale a $20 \mu \mathrm{m}$. Tomada con objetivo de 40X.

$$
\begin{aligned}
T_{i}= & \text { efecto del } i \text {-ésimo tratamiento } \\
E_{j(i)}= & \begin{array}{l}
\text { efecto de la } j \text {-ésima planta dentro del } i \text {-ésimo tratamiento (error } \\
\text { experimental) }
\end{array} \\
\delta_{k(i j)}= & \text { error de muestreo } \\
Y_{i j l}= & \begin{array}{l}
\text { observación en la } k \text {-ésima muestra, de la } j \text {-ésima planta, dentro del } \\
i \text {-ésimo tratamiento. }
\end{array}
\end{aligned}
$$

Con el fin de investigar la posible variación de origen ambiental, se utilizó un modelo adicional para detectarla entre plantas dentro de la línea AN-3, ya que ésta tiene un alto grado de homogeneidad genética. El modelo fue:

$$
Z_{i j}=u+P_{i}+\varepsilon_{j(i)}
$$

donde:

$$
\begin{aligned}
u & =\text { media general } \\
i & =1,2,15 \text { (plantas dentro de la línea AN-3) } \\
j & =1,2 \text { (muestras dentro de plantas) } \\
P_{i} & =\text { efecto de la i-ésima planta } \\
\varepsilon_{j(i)} & =\text { error de muestreo } \\
Z_{i j} & =\text { observación de la } j \text {-ésima muestra dentro de la } i \text {-ésima planta }
\end{aligned}
$$


Para investigar una posible asociación entre las observaciones de los cruzamientos con las plantas de los progenitores silvestres, se estimó el coeficiente de correlación lineal de Pearson y se probó de acuerdo con una distribución de $t$ de dos colas (Neter et al., 1989).

Todos los análisis numéricos se hicieron con porcentajes transformados con la función $\operatorname{ArSen} \sqrt{x / 100}$. Se utilizó R Development Core Team (2004) como software estadístico, con las funciones aov, y cor.test para análisis de varianza y de correlación, respectivamente.

\section{RESULTADOS Y DISCUSIÓN}

Los cruzamientos entre las ocho colectas de origen nativo y la línea CMSAN-3 produjeron un promedio de 71 aquenios bien desarrollados por capítulo. De las plantas de tales híbridos, 95\% produjeron polen, el cual tuvo una viabilidad de $94.6 \%$ (Cuadro 2). Lo anterior es una clara evidencia de la presencia de alelos restauradores de la fertilidad en las poblaciones silvestres estudiadas, debido a que todas las plantas de la generación $\mathrm{F}_{1}$ tenían citoplasma androestéril, que se hereda por vía materna, y la única manera de que produjeran polen es a través de genes nucleares restauradores. Por otro lado, uno de los individuos de procedencia espontánea presentó androesterilidad, lo cual indica la presencia de tal carácter en las poblaciones locales estudiadas. La androesterilidad en girasol se utiliza para la producción de híbridos de interés comercial, y todas las fuentes de citoplasma androestéril en esta especie provienen de girasoles silvestres (Jan, 2000). El hallazgo de nuevas fuentes de androesterilidad es importante para proveer diversidad al girasol cultivado.

Los porcentajes medios observados de tinción de polen fueron: 94.6 (error estándar $=0.90)$ para la línea AN-3, 95.2 (error estándar $=0.32)$ para el material silvestre (H. annuus ssp. texanus) y 91.5 (error estándar $=0.32$ ) para el híbrido (Cuadro 2). Para el caso del girasol cultivado Quillet et al. (1995) estimaron una viabilidad de $93 \%$ en la línea RHA274, porcentaje similar a lo obtenido en este trabajo. Para poblaciones silvestres de H. annuus, Jackson (1988) estimó 97.9\% de viabilidad de polen. Sin embargo, como se verá más adelante, parece haber un componente ambiental importante en la determinación de esta variable. Para el caso de los híbridos H. annuus var. macrocarpus x $H$. annuus ssp. texanus no se encontraron antecedentes en la literatura; sin embargo, se puede afirmar que la viabilidad media de polen observada es normal, dado que supera $90 \%$. En las investigaciones sobre tal carácter en cruzas amplias de girasol, este porcentaje se considera bastante aceptable (Jan, 2000). 
Cuadro 2. Porcentajes medios de viabilidad de polen (no transformada) en colectas de $H$. annuus ssp. texanus (HAT), en Helianthus annuus var. macrocarpus (HAM) y en los híbridos recíprocos (HIB).

\begin{tabular}{|c|c|c|c|}
\hline \multirow{2}{*}{ Colecta } & \multicolumn{3}{|c|}{ Viabilidad de Polen (\%) } \\
\cline { 2 - 4 } & HAT & HIB & HAM \\
\hline 1 & 97.8 & 58.3 & - \\
\hline 2 & 94.9 & 96.3 & - \\
\hline 3 & 86.1 & 96.3 & - \\
\hline 4 & 96.2 & 97.0 & - \\
\hline 5 & 96.1 & 88.7 & - \\
\hline 6 & 95.1 & 96.2 & - \\
\hline 7 & 97.6 & 95.4 & - \\
\hline 8 & 98.1 & 95.3 & - \\
\hline AN-3 & - & - & 94.6 \\
\hline Media & 95.2 & 91.5 & 94.6 \\
\hline Error estándar & 0.3 & 0.3 & 0.9 \\
\hline
\end{tabular}

Aunque se observaron divergencias numéricas entre taxa, entre colectas y entre cruzamientos, el análisis de varianza no reveló diferencias estadísticamente reveladoras para estos factores (Cuadro 3). Sin embargo, se encontraron discrepancias altamente significativas entre plantas dentro de taxa, de colectas y de cruzamiento. El análisis de varianza entre individuos dentro de la línea AN-3 también mostró diferencias de trascendencia, aun cuando esta línea por ser endogámica tiene un alto grado de homogeneidad genética. No se detectó correlación entre las viabilidades de polen en los híbridos y sus progenitores masculinos ( $r=-0.29 \mathrm{NS})$.

Los resultados indican que la variación en los materiales estudiados no obedece a la divergencia genética entre taxa, colonias o cruzamientos, sino a diferencias entre plantas dentro de los grupos. Estas disimilitudes significativas entre los individuos dentro de la línea AN-3 son indicativas de la presencia de una heterogeneidad ambiental que afecta a las plantas.

A la fecha no se ha encontrado una discrepancia marcada entre la viabilidad de polen de $H$. annuus cultivado y silvestre (Jackson, 1988, Quillet et al., 1995), lo cual puede explicarse como resultado de una fuerte presión selectiva hacia la retención de tal carácter, ya que este es un factor muy importante en la competencia reproductiva. 
Cuadro 3. Resultados de cuatro análisis de varianza para viabilidad de polen a través de tinción con Buffalo Black NBR. La simbología x/y representa x anidado en y. Taxa, colectas y cruzamientos se trataron como efectos fijos, mientras que las plantas individuales se consideraron como efectos aleatorios. En todos los casos el porcentaje de viabilidad de polen se transformó con la función $\operatorname{ArSen} \sqrt{x / 100}$.

\begin{tabular}{|l|c|l|}
\hline \multicolumn{1}{|c|}{ Comparación } & $\mathrm{F}$ & \multicolumn{1}{c|}{$\mathrm{P}$} \\
\hline Taxa & 2.03 & 0.16 \\
Plantas/Taxa & 2.43 & $0.00^{* *}$ \\
\hline Colecta & 2.18 & 0.09 \\
Plantas/Colecta & 8.69 & $0.00^{* *}$ \\
\hline Cruzamientos & 1.91 & 0.14 \\
Plantas/Cruzamientos & 27.91 & $0.00^{* *}$ \\
\hline Plantas/AN-3 & 5.88 & $0.00^{* *}$ \\
\hline
\end{tabular}

$\mathrm{F}=$ Valor del estadístico de prueba en el análisis de varianza.

$\mathrm{P}=$ Probabilidad del error Tipo I; $* *=\mathrm{P}<0.01$

La viabilidad de polen en los híbridos generados en este trabajo fue corroborada en forma cualitativa por medio de la germinación de polen así como por la capacidad de estos mismos híbridos de generar poblaciones $\mathrm{F}_{2}$ (Mendoza, 2003). Es de esperarse que en la mayoría de los casos la viabilidad de polen no sea una barrera para el uso de las poblaciones silvestres de Helianthus annuus procedentes de Saltillo y Ramos Arizpe, Coah., en programas de mejoramiento de girasol.

\section{CONCLUSIONES}

La viabilidad de polen en la línea AN-3 de girasol cultivado es similar a la de las poblaciones de Helianthus annuus ssp. texanus recolectadas en Saltillo, Coahuila. La domesticación del girasol no influyó considerablemente en este carácter, mismo que no diferencia a las poblaciones silvestres de las cultivadas.

La descendencia híbrida tuvo en promedio una viabilidad de polen que permite su reproducción sexual normal. De aquí que las poblaciones de $H$. annuus ssp. texanus sean una fuente viable para el manejo de infiltración de genes de materiales silvestres a cultivados. La producción de polen en $95 \%$ de las plantas híbridas es una evidencia clara de que las poblaciones silvestres que se manejaron en este trabajo poseen alelos para restauración de la fertilidad, los cuales pueden ser usados en 
programas de mejoramiento. Asimismo, el hecho de haber detectado una planta silvestre androestéril, convierte a las poblaciones estudiadas en posibles fuentes de esterilidad masculina. Por lo que respecta a las diferencias de viabilidad de polen entre plantas, éstas parecen obedecer principalmente a factores ambientales.

\section{AGRADECIMIENTOS}

Agradecemos al M. en C. Edgar Guzmán Medrano el habernos proporcionado materiales de la línea AN-3 de girasol cultivado utilizada en este trabajo, y a la Lic. Martha Gómez Martínez por la confección de la microfotografía de polen. Esta investigación fue financiada por el Consejo Nacional de Ciencia y Tecnología (Proyecto 26499-B) y por la Universidad Autónoma Agraria Antonio Narro (Proyecto 02-03-0203-2574).

\section{LITERATURA CITADA}

Alexander, M. P. 1969. Differential staining of aborted and non aborted pollen. Stain Technol. 44: 117-122.

Atlagic, J. 1996. Cytogenetic study in hexaploid Helianthus species and their $\mathrm{F}_{1}$ hybrids with cultivated sunflower, H. annuus. Plant Breeding 4: 257-260.

Atlagic, J., B. Dozet y D. Skoric. 1993. Meiosis and pollen viability in Helianthus tuberosus L. and its hybrids with cultivated sunflower. Plant Breeding 4: 318-324.

González-Elizondo, M. S. y D. Gómez-Sánchez. 1992. Notes on Helianthus (CompositaeHeliantheae) from Mexico. Phytologia 72: 63-70.

Gómez, D. y S. González. 1994. Localization of Helianthus, Viguiera and Tithonia genera in Mexico. In: Seiler, G. J. (ed.). FAO Working Group: Evaluation of wild Helianthus species. Progress Report 1991-1993: 134-150.

Jackson, R. C. 1988. A quantitative cytogenetic analysis of an intersectional hybrid in Helianthus (Compositae). Amer. J. Bot. 75: 219-222.

Jan, C. C. 2000. Cytoplasmic male sterility in two wild Helianthus annuus L. accessions and their fertility restoration. Crop Sci. 40: 1535-1538.

Mendoza, R. 2003. Huella de DNA, características morfológicas y viabilidad de polen en girasol cultivado y poblaciones silvestres de Helianthus annuus y Tithonia tubaeformis. Tesis doctoral. Universidad Autónoma Agraria Antonio Narro. Saltillo, Coah. 73 pp.

Neter, J., W. Wasserman y M. H. Kutner. 1989. Applied linear regression models. 2a. ed. Irwin. Boston, MA. $661 \mathrm{pp}$. 
Quillet, M. C., N. Madjidian, Y. Griveau, H. Serieys, M. Tersac, M. Lorieux y A. Bervillé. 1995. Mapping genetic factors controlling pollen viability in an interspecific cross in Helianthus sect. Helianthus. Theor. Appl. Genet. 91: 1195-1202.

R Development Core Team. 2004. R: a language and environment for statistical computing. R Foundation for Statistical Computing. Vienna.

Steel, R. G. y J. H. Torrie. 1960. Principles and procedures of statistics. McGraw-Hill Book Company, Inc. Nueva York. 481 pp.

Stelly, D. M., K. C. Kautz y W. L. Rooney. 1990. Pollen fertility of some simple and compound translocations of cotton. Crop Sci. 30: 952-955.

Whelan, E. D. P. 1982. Trisomic progeny from interspecific hybrids between Helianthus maximiliani and H. annuus. Canad. J. Genet. Cytol. 24: 375-384.

Recibido en mayo de 2003.

Aceptado en marzo de 2005. 